\title{
Diversidad del género Drosophila (Diptera, Drosophilidae) en el páramo de Papallacta, Pichincha, Ecuador
}

\author{
María Luna Figuero y Violeta Rafael \\ Laboratorio de Genética Evolutiva, Escuela de Ciencias Biológicas, Pontificia Universidad \\ Católica del Ecuador, Quito, Ecuador \\ lunaf3@hotmail.com; vrafael@puce.edu.ec
}

Recibido: 29, 01, 2013; aceptado: 18, 09, 2013

\begin{abstract}
RESUMEN.- El presente estudio se realizó en bosques de Polylepis situados entre los 3700 y $4000 \mathrm{~m}$ de altitud. Estos ecosistemas se encuentran amenazados por la fragmentación debido a la deforestación. Cuando la fragmentación aumenta y la cantidad de hábitat remanente disminuye, se reduce la diversidad del ecosistema. Fueron seleccionados tres parches de bosque de Polylepis. En cada parche se estableció un transecto de $120 \mathrm{~m}$ de longitud dividido en siete estaciones de muestreo. En total se capturaron 572 individuos pertenecientes a ocho especies del género Drosophila. Uno de los parches presentó la mayor abundancia y la mayor diversidad de drosófilas, además de ser el más diverso en flora. No se capturaron especies cosmopolitas en ninguno de los parches, lo cual indicó que los bosques se encuentran en buen estado de conservación; sin embargo, una posible explicación podría ser que las bajas temperaturas del páramo estén actuando como barrera.
\end{abstract}

PALABRAS CLAVES: biodiversidad, bioindicador, Drosophila, fragmentación, páramo

\begin{abstract}
This study was conducted in forests of Polylepis located between 3700 and $4000 \mathrm{~m}$ above sea level. These ecosystems are threatened by fragmentation from deforestation. As fragmentation increases and the amount of remaining habitat decreases, the ecosystem diversity may be reduced. We selected three forest patches of Polylepis. In each patch we established a transect of $120 \mathrm{~m}$ divided into seven sampling sites. We captured 572 individuals belonging to eight species of the genus Drosophila. One of the patches had the highest abundance and greater diversity of fruit flies and plants. We did not capture cosmopolitan species in any of the patches, indicating that these forests are in good condition. However an alternative explanation could be that the low temperatures of the páramo are acting as a barrier to dispersal of cosmopolitan species from lower elevations and other locations.
\end{abstract}

KEYWORDS: biodiversity, bioindicator, Drosophila, fragmentation, páramo 


\section{INTRODUCCIÓN}

La familia Drosophilidae está compuesta por 73 géneros y 3950 especies descritas y distribuidas en las regiones templadas y tropicales del planeta (Brake y Bächli, 2008). En la región Neotropical, el género Drosophila está altamente diversificado, con numerosas especies aún por descubrirse (Medeiros y Klaczko, 2004). Las investigaciones sobre la biodiversidad de la flora y fauna en Ecuador, han revelado que a pesar de ser un país relativamente pequeño, es biológicamente uno de los más diversos del planeta (Mittermeier y Mittermeier, 1997).

La primera referencia sobre el género Drosophila en el Ecuador fue el estudio de Becker (1919). Sin embargo, investigaciones minuciosas sobre la diversidad del género se iniciaron solo a finales de la década de los años 80. Durante los últimos 20 años se han obtenido datos sobre la distribución y taxonomía del género, con inclusión de las descripciones de especies nuevas (ie. Acurio et al., 2010; Acurio y Rafael, 2009; Vela y Rafael, 2005; Rafael y Vela, 2000). Hasta el 2009, en el Ecuador, se han reportado 112 especies del género (Acurio y Rafael, 2009). En estudios recientes (Céspedes y Rafael, 2012; Figuero et al., 2012; Figuero y Rafael, 2011) se descubren 8 nuevas especies más del género Drosophila.

A pesar que el género Drosophila ha sido utilizado como organismo modelo en innumerables publicaciones (Mateus et al., 2006), pocas de estas han abordado el ámbito de la ecología y la distribución geográfica; en la actualidad existe un mayor interés en los estudios ecológicos mediante Drosophila; por ejemplo varios autores han propuesto al género Drosophila como bioindicador (Cavasini et al., 2008; Alves da Mata et al., 2008; Gottschalk et al., 2007; Tidon, 2006; Ferreira y Tidon, 2005; Van der Linde y Sevenster, 2002).

El presente estudio se realizó en tres parches de bosques de Polylepis con influencia antrópica. Polylepis es una planta leñosa de la familia Rosaceae con distribución sobre los $3500 \mathrm{~m}$ de altitud. Estos bosques cumplen un papel central en la ecología alto-andina, como hábitat de muchas especies de plantas y animales (Kessler, 2006). Sin embargo, estos bosques son uno de los ecosistemas más amenazados del mundo. La principal amenaza es la fragmentación por deforestación. La mayoría de los bosques andinos tropicales se caracterizan por presentar una distribución en parches; las posibles causas de esta distribución están aún en debate (Cierjacks et al., 2007). Cuando la fragmentación aumenta y la cantidad de hábitat remanente disminuye, las poblaciones animales se reducen por la emigración de los individuos o por un aumento en la mortalidad que afectan la diversidad del ecosistema (Grez y Zaviezo, 2002; Bustamante y Grez, 1995).

El objetivo de este trabajo fue analizar la diversidad del género Drosophila y el estado de conservación en que se encuentran los parches mediante la ausencia o presencia de ciertas especies del género Drosophila. 


\section{MATERIALES Y MÉTODOS}

Fueron seleccionados tres parches de bosques de Polylepis (denominados parche A, B y C), en la vía Pifo-Páramo de Papallacta de la provincia de Pichincha, Ecuador (Figura 1). Estos parches se componen principalmente de árboles del género Polylepis, y forman parte de la Reserva Ecológica Cayambe-Coca y la Reserva Ecológica Antisana; ubicadas en la parte occidental de la cordillera oriental.

El parche A es un remanente ubicado a $3731 \mathrm{~m}$ de altitud, cuyas coordenadas son 78¹3'28.1'”; 00¹9'13.5'S. Este parche es un relicto boscoso homogéneo de Polylepis incana Kunth, en cuyo interior se encuentran numerosas matas de Gynoxys sp., una planta arbustiva de la familia Asteraceae.

El parche B se sitúa a $4014 \mathrm{~m}$ de altitud, con coordenadas $78^{\circ} 12^{\prime} 32.1^{\prime \prime} \mathrm{W}$; 00²0'09.4"S. Estebosque tiene composición mixta de plantas de páramo, en su mayoría formadas por Polylepis pauta Hieron; además se encuentra una gran variedad de especies de la familia Asteraceae.

El parche C se encuentra a $4005 \mathrm{~m}$ de altitud, sus coordenadas son $78^{\circ} 12^{\prime} 42.8^{\prime \prime} \mathrm{W}$ $00^{\circ} 19^{\prime} 24.8^{\prime \prime}$ S. Este relicto boscoso presenta una composición homogénea de Polylepis pauta, aunque en las cercanías podemos encontrar grandes poblaciones de Gynoxis sp.
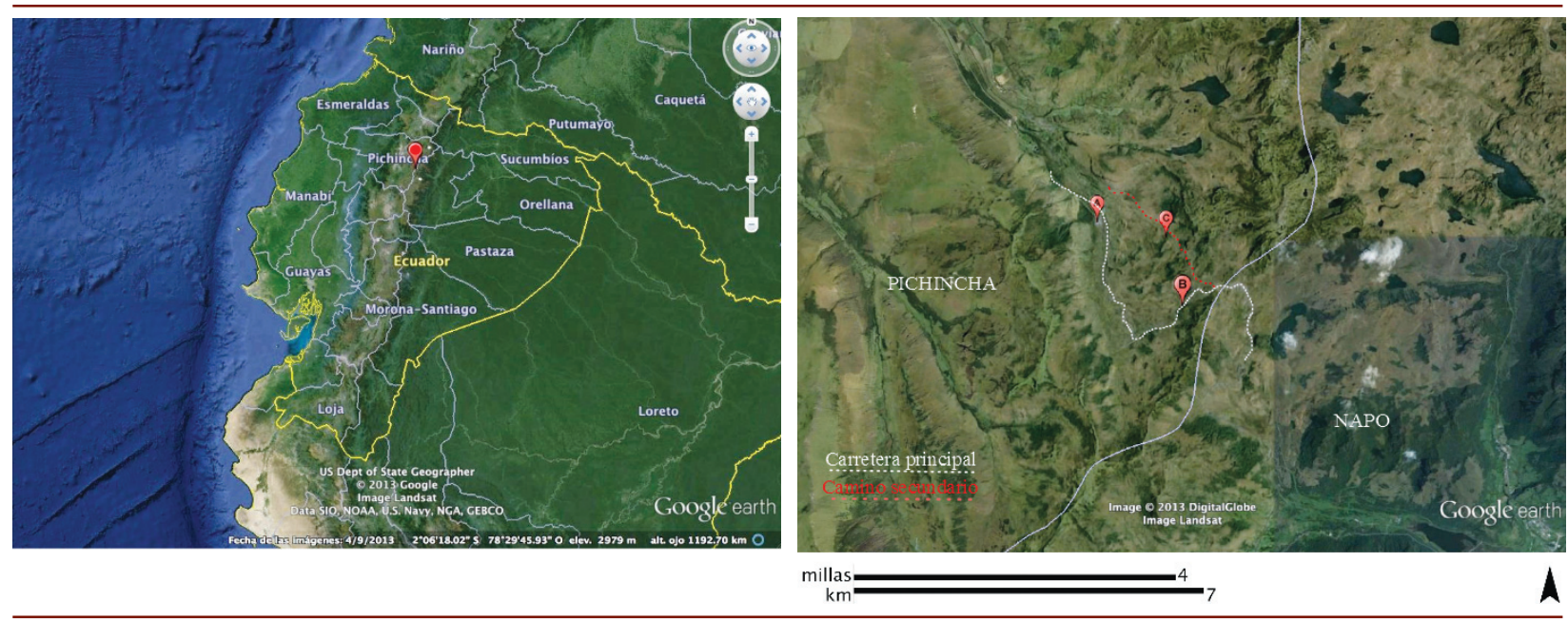

Figura 1. Mapa y ubicación de los tres parches muestreados durante el estudio. 
En cada parche se estableció un transecto de $120 \mathrm{~m}$ de longitud, desde el borde del parche hacia el interior del bosque, dividido en siete estaciones de muestreo separadas por una distancia de 20 m. En cada estación se colocaron cuatro trampas que contenían cebo de plátano y levadura. Las trampas fueron fabricadas con botellas plásticas perforadas para facilitar el ingreso de las moscas. Las trampas fueron atadas en árboles a $1 \mathrm{~m}$ de altura. Las recolecciones se realizaron con aspiradores entomológicos durante los meses de febrero, abril, julio y septiembre del 2009.

Se calculó la diversidad beta de Whittaker en cada parche que nos indica la tasa de cambio de especies de Drosophila entre los tres parches. Se calculó con la siguiente fórmula;

$$
\beta_{\mathrm{w}}=(\mathrm{S} / \alpha)-1
$$

donde $S=$ número total de especies encontradas en el sistema (diversidad gama) y $\alpha=$ el promedio de la diversidad encontrada (Magurran, 2004).

La identificación de las especies del género Drosophila se hizo mediante el análisis de la morfología externa y de la genitalia. Los individuos de las especies nuevas descritas previamente se depositaron en el Museo de Zoología, sección Invertebrados de la Pontificia Universidad Católica del Ecuador, Quito (QCAZ-I).

Por último, se realizó una caracterización de hábitat, para ello se colectaron las plantas de cada estación de muestreo de cada transecto en los tres parches. La identificación de las muestras se realizó con la ayuda de especialistas del Herbario QCA de la Pontificia Universidad Católica del Ecuador.

\section{RESULTADOS}

Diversidad.- Se capturaron 572 individuos pertenecientes a ocho especies del género Drosophila y dos especies de hembras no determinadas (Tabla 1). Cinco de las especies corresponden a especies nuevas; tres de ellas ya descritas (Figuero et al., 2012; Figueroy Rafael, 2011), Drosophila papallacta sp. nov., (en preparación) y Drosophila $1 \mathrm{sp}$. nov. sin publicar.

En el parche A se capturaron 64 individuos pertenecientes a cuatro especies; en el parche B se recolectaron 386 individuos, miembros de ocho especies y en el parche $\mathrm{C}$ se encontraron 122 individuos pertenecientes a tres especies del género Drosophila (Tabla 1). El parche A presentó una diversidad $\beta_{\mathrm{w}=} 0.86$, el parche $\mathrm{B}, \beta_{\mathrm{w}=} 1 \mathrm{y}$ el parche $C, \beta_{w=} 0.62$.

Las ocho especies capturadas son del subgénero Drosophila y pertenecen a seis grupos de especies menos una especie no agrupada. Grupo Drosophila guarani representada por $D$. ecuatoriana Vela $y$ Rafael, 2004; grupo D. tripunctata por D. pasochoensis Vela y Rafael, 2001; grupo D. mesophragmatica por D. rucux Céspedes y Rafael, 2012; grupo D. flavopilosa por Drosophila $1 \mathrm{sp}$. nov.; grupo D. onychophora por Drosophila yurag y Drosophila yuragshina 
Figuero y Rafael, 2011; grupo D. asiri por sin agrupar: Drosophila papallacta sp. nov. Drosophila yanaurcus Figuero et al. 2012 y (en preparación).

Tabla 1

Especies del género Drosophila recolectadas en cada parche y en cada una de las siete estaciones del transecto

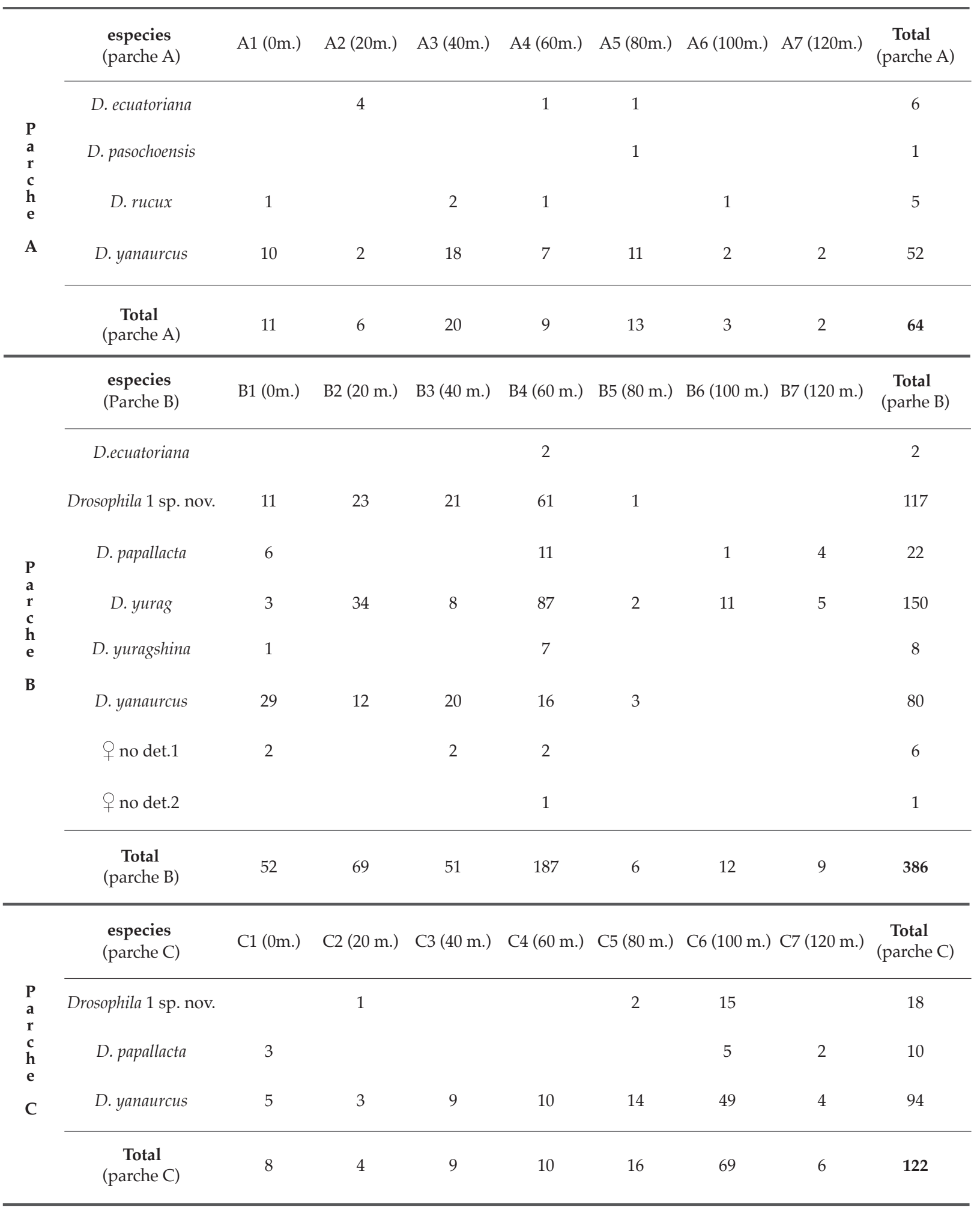


Los resultados de la diversidad parche $\mathrm{B}$ fue el más diverso en plantas, vegetal son los siguientes: en el parche A se colectaron 37 especies, 18 de ellas de se colectaron un total de 25 especies de la familia Asteraceae. En el parche $C$ se plantas a lo largo de todo el transecto, en colectaron 29 especies de plantas, también su mayoría de la familia Asteraceae. El es su mayoría asteráceas (Tabla 2).

Tabla 2

Especies y porcentajes de plantas encontradas en cada uno de los parches muestreados

\begin{tabular}{|c|c|c|c|c|c|}
\hline especies parche $A$ & $\%$ & especies parche $B$ & $\%$ & especies parche $C$ & $\%$ \\
\hline Aetheoleanae sp. & 15 & ASTERACEAE sp.3 & 0,28 & ASTERACEAE sp.1 & 1,14 \\
\hline ASTERACEAE sp.1 & 7,14 & ASTERACEAE sp.4 & 1,14 & ASTERACEAE sp.2 & 0,71 \\
\hline ASTERACEAE sp.2 & 1,42 & ASTERACEAE sp.6 & 1,14 & ASTERACEAE sp.3 & 4,28 \\
\hline ASTERACEAE sp.3 & 0,42 & ASTERACEAE sp.7 & 0,57 & ASTERACEAE sp.4 & 1,85 \\
\hline ASTERACEAE sp.4 & 0,14 & ASTERACEAE sp. 8 & 0,28 & ASTERACEAE sp.5 & 1,42 \\
\hline ASTERACEAE sp.5 & 6,14 & Azorella sp. & 1 & ASTERACEAE sp. 8 & 0,14 \\
\hline Butleja incana & 2 & Baccharis sp. & 0,57 & Azorella sp. & 0,57 \\
\hline Calamagrostis intermedia & 0,14 & Calamagrsotis intermedia & 2 & Carex pichinchensis & 0,14 \\
\hline Gunera magenalica & 1,14 & Daucus sp. & 0,14 & Diplostephium sp.1 & 0,42 \\
\hline Hydrocotyle sp. & 3,28 & Diplostephium rupestre & 0,57 & ERICACEAE sp.1 & 1 \\
\hline Hypericum laricifolium & 0,14 & Diplostephium sp.1 & 3,14 & Galium sp. & 0,14 \\
\hline Lachemila orbiculata & 13,57 & Diplostephium sp.2 & 6,85 & Gynoxis sp.1 & 0,57 \\
\hline Lachemila sp. & 0,85 & Diplostephium sp.3 & 1,57 & Gynoxis sp.2 & 3,71 \\
\hline Luzula gigantea & 0,71 & Galium canescens & 2 & Hydrocotyle sp. & 14 \\
\hline Pernettya postrata & 0,14 & Geranium diffusum & 3,42 & Luzula gigantea & 0,71 \\
\hline Polylepis incana & 28 & Gunnera magellanica & 8,14 & Miconia sp.1 & 4,42 \\
\hline Polylepis pauta & 0,85 & Gynoxis monophylla & 1 & Miconia sp.2 & 2,57 \\
\hline Ribes sp. & 3,14 & Gynoxis sp.2 & 1,85 & Monticalia andicola & 1,57 \\
\hline ROSACEAE sp.1 & 0,71 & Gynoxis sp.3 & 1,85 & Polylepis pauta & 20,71 \\
\hline Sibthorpia repenes & 5,42 & Gynoxis sp.4 & 1,42 & Rubus sp. & 0,71 \\
\hline Solanum sp.1 & 4,42 & Gynoxis sp.5 & 2,57 & Sibthorpia repenes & 33 \\
\hline Solanum sp.2 & 0,57 & Gynoxis sp.6 & 2,57 & Solanum sp.1 & 1,42 \\
\hline Urtica sp.1 & 1 & Loricaria thuyoides & 0,42 & Solanum sp.2 & 2 \\
\hline Urtica sp.2 & 1,57 & Luzula gigantea & 2,85 & Solanum sp.3 & 1,42 \\
\hline \multirow[t]{13}{*}{ Urtica sp.3 } & 2 & Miconia sp.1 & 7 & Solanum sp.4 & 0,28 \\
\hline & & Monina sp. & 0,42 & Solanum sp.5 & 0,28 \\
\hline & & Pernettya sp. & 0,42 & Stellaria sp. & 0,14 \\
\hline & & Polylepis pauta & 27,57 & Urtica sp.2 & 0,28 \\
\hline & & Rubus sp. & 1,57 & nodet. & 0,28 \\
\hline & & Sibthorpia repens & 0,28 & & \\
\hline & & Solanum sp.3 & 0,57 & & \\
\hline & & Solanum sp.5 & 1,42 & & \\
\hline & & Stellaria sp. & 1,14 & & \\
\hline & & Valeriana microphylla & 5,14 & & \\
\hline & & Valeriana sp. & 6,42 & & \\
\hline & & Werneria sp. & 0,28 & & \\
\hline & & nodet. & 0,28 & & \\
\hline \multicolumn{6}{|l|}{ Especies más abundantes } \\
\hline \multicolumn{2}{|c|}{ Especies de la familia ASTERACEAE } & & & & \\
\hline
\end{tabular}


Distribución temporal de las En este parche se observa una pequeña especies.- En el parche A, abril fue el mes distribución temporal de las especies más con más abundancia de individuos y más abundantes (Tabla 3, Figura 3).

diversidad, mientras que julio fue el mes de menor captura de individuos (Tabla 3, Figura 2).

En el parche $C$, al igual que en el parche $B$, febrero fue el mes en el cual se recolectó el mayor número de individuos,

EnelparcheB, enfebrerosecapturaron y abril fue el mes en el cual se encontró más individuos y más especies (8 especies); mayor diversidad, mientras que en julio mientras que julio sigue siendo el mes con se capturó el menor número de individuos menor número de individuos capturados. (Tabla 3, Figura 4).

Tabla 3

Especies del género Drosophila recolectadas en cada parche y en cada mes de muestreo. *mes de mayor captura

\begin{tabular}{|c|c|c|c|c|c|c|}
\hline & Especie & Feb. & Abr. & Jul. & Sep. & SubTotal \\
\hline \multirow{4}{*}{ Parche A } & D. yanaurcus & 11 & 24 & 2 & 15 & 52 \\
\hline & D. rucux & & 1 & 1 & 3 & 5 \\
\hline & D. pasochoensis & & 1 & & & 1 \\
\hline & D. ecuatoriana & 5 & 1 & & & 6 \\
\hline & Subtotal Parche A & 16 & $* 27$ & 3 & 18 & 64 \\
\hline \multirow{8}{*}{ Parche B } & D. yanaurcus & 38 & 8 & 10 & 24 & 80 \\
\hline & D. yuragshina & 1 & 4 & & 3 & 8 \\
\hline & D. yurag & 24 & 56 & 9 & 61 & 150 \\
\hline & D. papallacta & 2 & 8 & 5 & 7 & 22 \\
\hline & Drosophila 1 sp. nov & 94 & 10 & 12 & 1 & 117 \\
\hline & D. ecuatoriana & 1 & & & 1 & 2 \\
\hline & nodet.2 & & & & 1 & 1 \\
\hline & nodet.1 & & & 1 & 5 & 6 \\
\hline & Subtotal Parche B & *160 & 86 & 37 & 103 & 386 \\
\hline \multirow{3}{*}{ Parche C } & D. yanaurcus & 29 & 33 & 1 & 31 & 94 \\
\hline & D. papallacta & & 1 & 2 & 7 & 10 \\
\hline & Drosophila 1 sp. nov & 15 & 3 & & & 18 \\
\hline & Subtotal Parche C & ${ }^{*} 44$ & 37 & 3 & 38 & 122 \\
\hline & TOTAL & 220 & 150 & 43 & 159 & 572 \\
\hline
\end{tabular}


|| Revista Ecuatoriana de

IEDICINA Y CIENCIAS BIOLÓGICAS

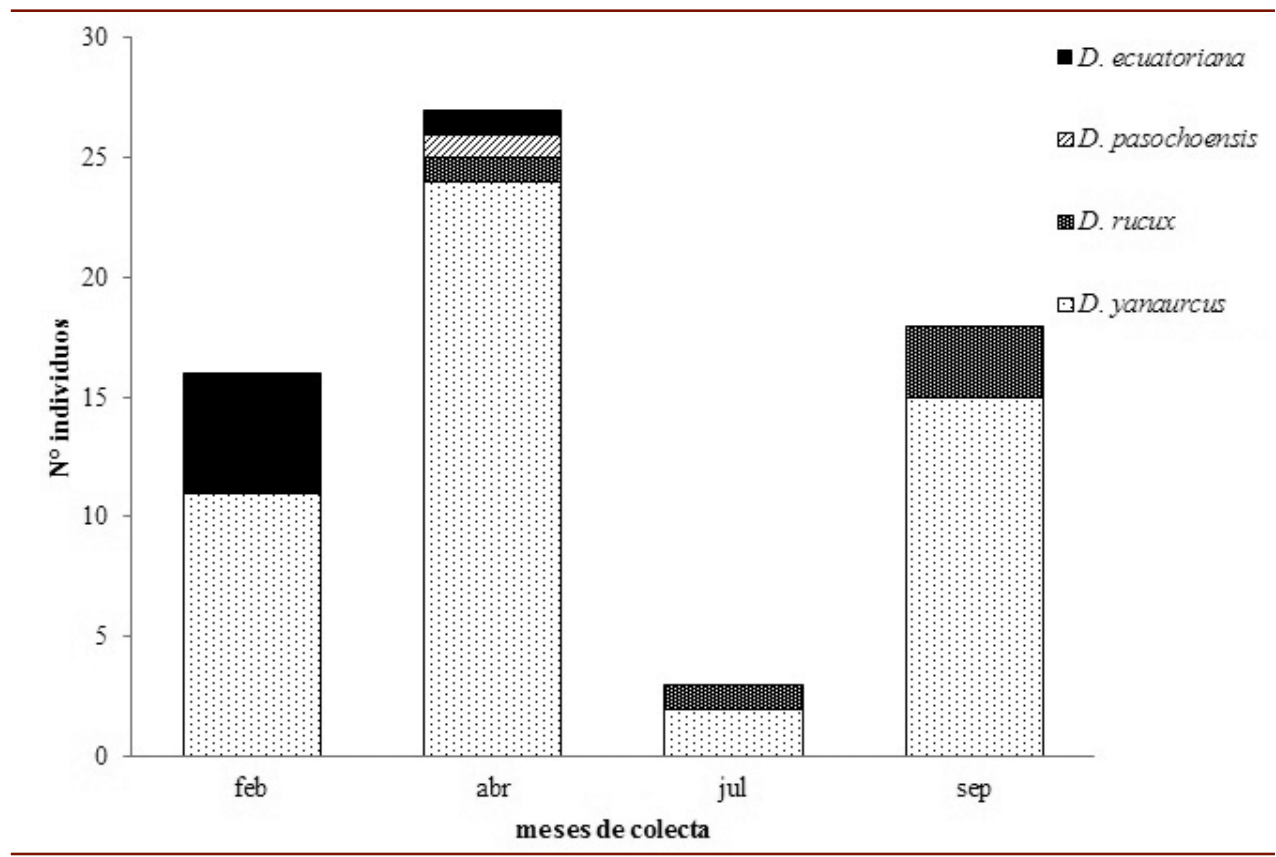

Figura 2. Diversidad y abundancia de las especies del género Drosophila en el parche A, durante los cuatro meses de colecta.

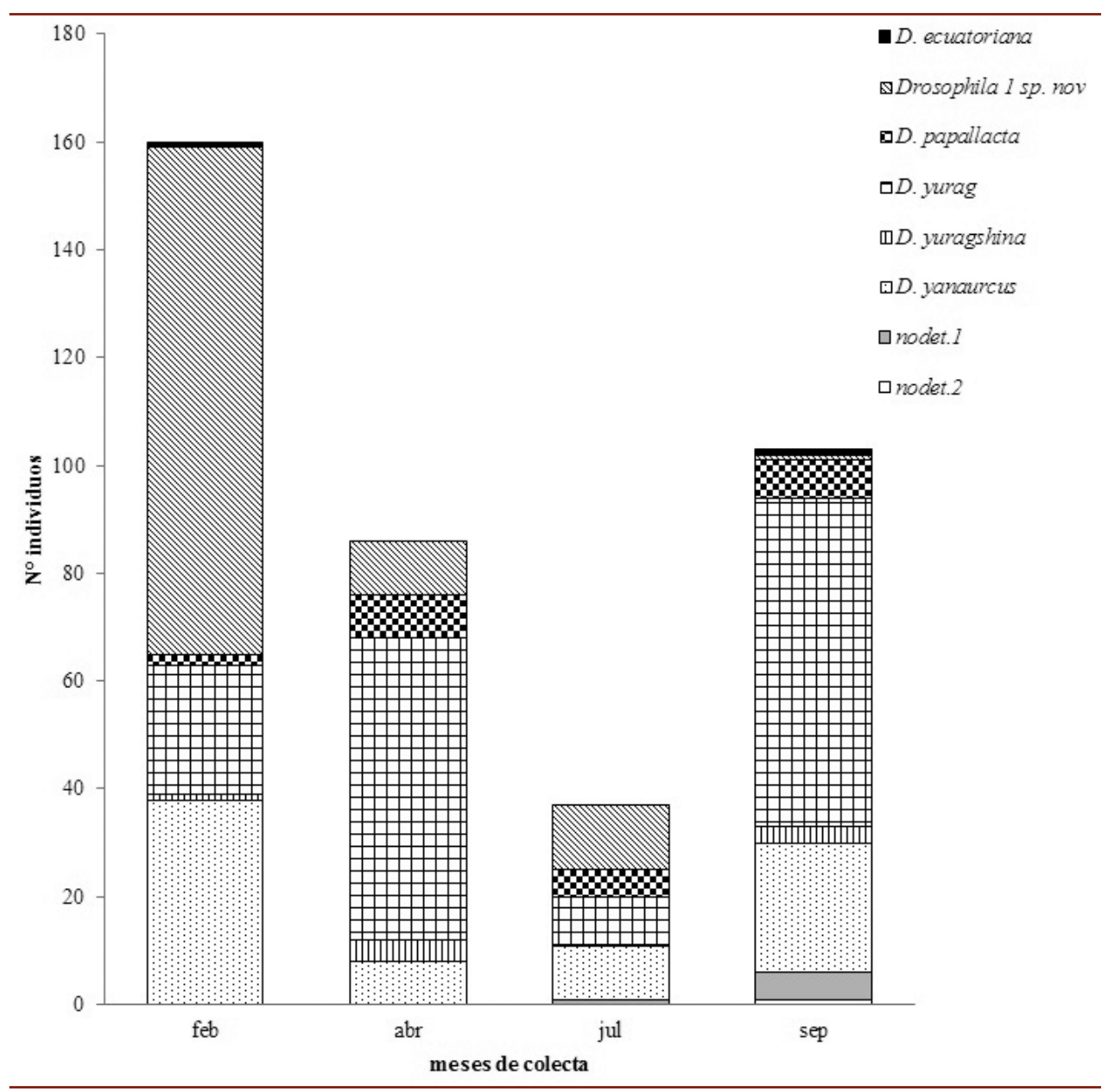

Figura 3. Diversidad y abundancia de las especies del género Drosophila en el parche B, durante los cuatro meses de colecta. 


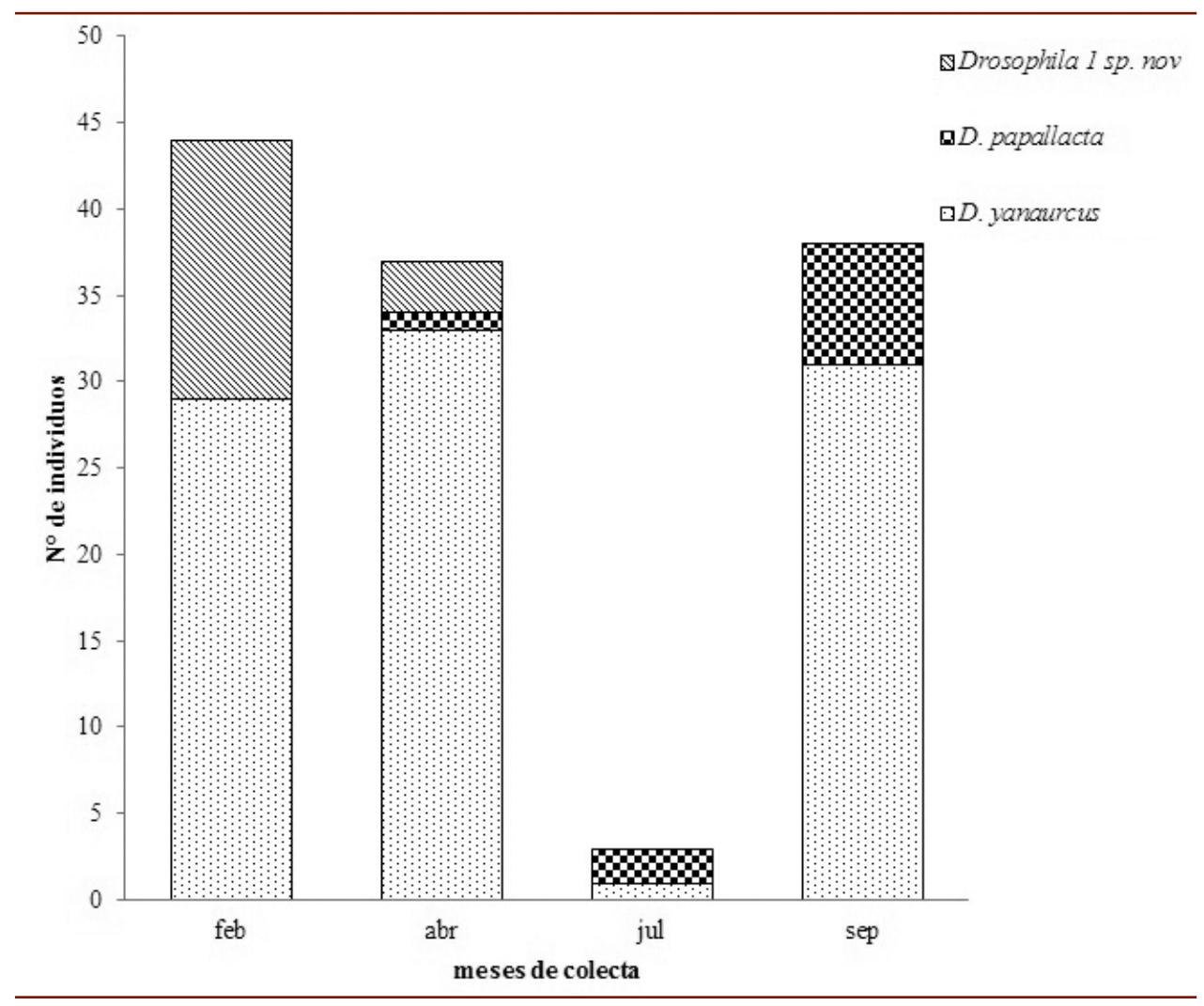

Figura 4. Diversidad y abundancia de las especies del género Drosophila en el parche C, durante los cuatro meses de colecta.

\section{DISCUSIÓN}

Composición de las comunidades del género Drosophila y caracterización de hábitat.- El número de moscas capturadas en este páramo es aparentemente bajo (572 individuos), en comparación con otros estudios realizados en los últimos años en el Ecuador (Acurio y Rafael, 2009; Rafael y Vela, 2000). Hay que resaltar que los bosques muestreados en el presente trabajo se encuentran sobre los $3700 \mathrm{~m}$ de altitud. A esta misma altitud en la Quebrada de Cruz Loma, Pichincha, Céspedes y Rafael encontraron un total de 11 individuos del género Drosophila, mientras que a $4000 \mathrm{~m}$ de altitud en la misma quebrada no capturaron ningún individuo (com. pers. Céspedes, 2011). Por lo tanto se debe considerar que el muestreo realizado en este estudio fue óptimo. Además, en estos bosques las condiciones ambientales son extremas, con temperaturas máximas de hasta $20^{\circ} \mathrm{C}$ (entre las 12:00 y 15:00 h) y mínimas de $-2^{\circ}$ C (entre las 3:00 y 6:00 h). Las variaciones de temperatura extremas tienen efectos perjudiciales en los ectotermos como los insectos (Dillon et al., 2009) por lo cual encontrar especies de Drosophila en estos lugares resulta extraordinario.

De las ocho especies de Drosophila encontradas, cinco son nuevas especies. Tres 
ya han sido descritas (Figuero et al., 2012; Figuero y Rafael, 2011); D. papallacta sp. nov. se encuentra en preparación, mientras que Drosophila 1 sp. nov. (grupo D. flavopilosa) aún no se ha publicado debido a que es una especie muy similar a $D$. korefae Vela y Rafael, 2004. La única diferencia que existe entre las dos especies es que Drosophila 1 sp. nov. presenta pigmentación en el ala, mientras que $D$. korefae no la presenta (obs. pers.); esta diferencia no es suficiente para aseverar que se trata de especies diferentes, por lo cual se espera en el futuro se realicen análisis moleculares.

En conclusión, estos descubrimientos indicarían la existencia de numerosas especies nuevas del género Drosophila en los bosques de Polylepis y del alto endemismo que potencialmente pueden existir en los páramos del Ecuador. Por consiguiente se deben considerar como prioritarios a estos ecosistemas para futuros estudios de diversidad.

Drosophila yanaurcus se encuentra distribuida en los tres parches, mientras que el parche B y C comparten a Drosophila 1 sp. nov., y Drosophila papallacta sp. nov. (en preparación). Estos resultados estarían sugiriendo que las tres especies están mejor adaptadas a los ecosistemas de altura como son los bosques de Polylepis que el resto de especies encontradas. Sin embargo, ¿Cómo se explicaría que los tres parches muestreadoscompartenlasmismasespecies de Drosophila si se conoce que las moscas de altura seguramente tengan poca capacidad de vuelo? (Dillon y Frazier, 2006; obs. pers.)
Aragundi y colaboradores (2010); Kessler (2002) y Ellenberg (1979) sugieren que los bosques de Polylepis, cuando aparecieron, formaban bosques continuos y no parches como los conocemos en la actualidad, lo cual explicaría en parte la presencia de las mismas especies del género Drosophila en varios parches.

Los resultados de la caracterización de hábitat muestran que el parche B es más diverso en plantas que los otros dos parches ( $\mathrm{A}=25$ especies de plantas; $\mathrm{B}=37$ especies $\mathrm{y}$ $\mathrm{C}=29$ especies). La alta diversidad vegetal del parche B podría ser considerada como una fuente nutricional importante para las drosófilas, lo cual explicaría la presencia de las ocho especies del género Drosophila. Dos de las especies de Drosophila pertenecen al grupo de especies D. onychophora, grupo asociado a flores de la familia Asteraceae (Figuero y Rafael, 2011), familia abundante en el parche B. Asimismo este parche presentó la diversidad beta más alta $\left(\beta_{\mathrm{w}}\right.$ $(\mathrm{de} B)=1$ ) en comparación con los parches A y C. Esto nos indica que el parche B es el más heterogéneo en cuanto a la comunidad de especies de Drosophila; por lo tanto este relicto boscoso tan diverso debe ser prioritario en programas de conservación.

Distribución temporal.- Febrero fue el mes donde se recolectó el mayor número de individuos (220), mientras que julio fue el mes con menor número de individuos recolectados. Los datos del INAMHI, de la estación AMAZNOR (Papallacta), para el 2009 indican al mes de febrero como uno de los más secos $(57.3 \mathrm{~mm})$ mientras que 
julio es uno de los meses más lluvioso del año (155.2 mm) (INAMHI, 2009), además de ser un mes con vientos fuertes (obs. pers.), estas variaciones ambientales pueden ser la causa que se recolectaron más individuos de ciertas especies que otras según el mes, lo cual indicaría una distribución temporal de las poblaciones de las diferentes especies. Por ejemplo Drosophila 1 sp. nov., es abundante en el parche $\mathrm{B}$ y $\mathrm{C}$ en febrero mientras que casi no se encuentra en septiembre. Otro caso es Drosophila yurag que se encuentra en el parche B; esta especie declina su población en el mes de julio y se recupera en septiembre. Por último, Drosophila yanaurcus disminuye su población en julio en todos los parches, pero reaparece en septiembre. Los meses en que declinan las poblaciones podrían corresponder a estadios larva/ pupa de las especies. Las moscas de altura presentan ciclos de vida largos debido a las bajas temperaturas (aproximadamente 3 meses), por ejemplo D. yanaurucs (Figuero et al., 2012). Existen otros estudios sobre los ciclos biológicos de especies del género Drosophila en relación con la temperatura (James et al., 1997; Shorrocks, 1972). Estos estudios permiten afirmar que las moscas de altura se pueden encontrar en estadios de larva/ pupa durante tres meses. Este tiempo podría corresponder a las épocas en que las poblaciones de Drosophila de los parches A, B y C de los bosques de Polylepis decrecen.

Estado de conservación de los bosques.- La abundancia y composición de la población de un determinado taxón puede indicarnos la calidad de un ecosistema. Existen grupos de insectos bioindicadores del estado de conservación de los ecosistemas, como las mariposas, las hormigas y los macroinvertebrados entre otros (Bonada et al., 2006; Meyer y Sisk, 2001; Andersen, 1997). Las especies cosmopolitas del género Drosophila, como algunos miembros del grupo $D$. melanogaster, indican alteraciones en el ecosistema y podrían estar favorecidas por la fragmentación, mientras que las endémicas, indican la existencia de factores ecológicos excepcionales y un ecosistema único (Vela y Rafael, 2003). Al contrario de los resultados obtenidos por Acurio y colaboradores (2010), quienes encuentran una elevada tasa de drosófilas invasoras en lugares con influencia antrópica; en el Bosque Tropical Yasuní (Ecuador), en el presente estudio no se encontró ninguna especie del género Drosophila en calidad de cosmopolita en los bordes de los parches de Polylepis, a pesar de su cercanía a lugares con actividad antrópica. Estos resultados son alentadores en cuanto al estado de conservación de los bosques de Polylepis. Una de las razones principales para que no se hayan encontrado especies cosmopolitas puede ser las bajas temperaturas del páramo. Existen estudios que confirman la poca adaptabilidad de las especies cosmopolitas a las bajas temperaturas, como es el caso de D. melanogaster Meigen, 1830 (Hoffmann, 2010; Dillon y Frazier, 2006). Otro ejemplo es el caso de D. malerkotliana Parshad y Paika, 1965, especie del grupo D. melanogaster, que fue capturada en el parche B en el año 2004 (Rafael, 2007). Durante el presente estudio no se capturó ningún individuo de esta especie; esto corroboraría 
que las poblaciones de D. malerkotliana llegaron al páramo accidentalmente pero no se establecieron, probablemente debido a las bajas temperaturas del lugar. Por lo tanto, en el caso de los bosques de altura, las especies cosmopolitas no serían buenas indicadoras de disturbio o intervención. Así mismo, existe la posibilidad que las especies encontradas en el presente estudio no sean endémicas o nativas de los lugares de colecta, por lo cual sugerimos estudios a largo plazo para evaluar los efectos de la fragmentación en los bosques de Polylepis.

\section{AGRADECIMIENTOS}

A la Pontifica Universidad Católica del Ecuador por financiar los proyectos: "Caracterización de nuevas especies ecuatorianas de Drosophila" No. E29050 y "Diversidad del género Drosophila en bosques de Polylepis de la provincia de Pichincha" No. E29181. Al Ministerio de Ambiente por emitir la autorización de investigación científica No. 008-09 IC-FAUDNB / MA. Al Dr. Carlos Ribeiro Vilela de la Universidad de São Paulo, por sus valiosos comentarios. Al licenciado Juan Iglesias por la ayuda con la identificación de las plantas. A Katya Romoleroux Ph.D por la información brindada sobre los bosques de Polylepis. A nuestros colegas Olivier Dangles Ph.D y Rommel Montúfar Ph.D, por revisar y comentar este manuscrito. Al personal del laboratorio de Genética Evolutiva y a la licenciada Fernanda Salazar de la PUCE. A Idea Wild por la donación de materiales.

\section{REFERENCIAS BIBLIOGRÁFICAS}

Acurio A, Rafael V y Dangles O. 2010. Biological Invasions in the Amazonian Tropical Rain Forest: The Case of Drosophilidae (Insecta, Diptera) in Ecuador, South America. Biotropica, DOI:10.1111/j.1744-7429: 1-7.

Acurio AE y Rafael VL. 2009. Inventario taxonómico de Drosophilidae (Diptera) en el parque Nacional Yasuní, Amazonía Ecuatoriana. Acta Amazonica, 39(3): 713-718.

Alves da Mata R, McGeoch M y Tidon R. 2008. Drosophilid assemblages as a bioindicator system of human disturbance in the Brazilian Savanna. Biodiversity and Conservation, 17: 2899-2916.

Andersen AN. 1997. Using Ants as bioindicators: Multiscale Issues in Ant Community Ecology. Conservation Ecology, 1(1), 8. Página de Internet: http:// www.consecol.org/vol1/iss1/art8 / Consultada diciembre-2012.

Aragundi S, Hamrick JL y Parker KC. 2010. Genetic insights into the historical distribution of Polylepis pauta (Rosaceae) in the northeastern Cordillera Oriental of Ecuador. Conservation Genetics, DOI:10.1007/ s10592-010-0165-x

Becker T. 1919. Diptères brachycères. Mission du service géographique de l'armée. Mesure d'un arc de méridien équatorial 
en Amérique du sud, 10(2): 143-211.

Bonada N, Prat N, Resh V y Statzner B. 2006. Developments in aquatic insect biomonitoring: A comparative analysis of recent approaches. Annual Review of Entomology, 51: 495-523.

Brake I y Bächli G. 2008. World catalogue of insects. Volume 9. Drosophilidae (Diptera). Apollo Books. Stenstrup, Denmark. 412 pp.

Bustamante R y Grez AA. 1995. Consecuencias ecológicas de la fragmentación de los bosques nativos. Ambiente y desarrollo, 11(2): 58-63.

Cavasini R, Mateus RP y Tunes BML. 2008. Caracterização da assembléia de Drosophila (Diptera, Drosophilidae) em dois fragmentos florestais na região sul do Brasil. Revista Eletrônica Lato Sensu, 3(1). Página de Internet: http: / www.unicentro.br. Consultada diciembre-2012.

Céspedes D y Rafael V. 2012. Cuatro especies nuevas del grupo de especies Drosophila mesophragmatica (Diptera, Drosophilidae) de Los Andes ecuatorianos. Iheringia, Série Zoologia, 102(1): 71-79.

Cierjacks A, Wesche K y Hensen I. 2007. Potential lateral expansion of Polylepis forest fragments in central Ecuador. Forest Ecology and Management, 242: 477-486.
Dillon M, Wang G, Garrity P y Huey R. 2009. Thermal preference in Drosophila. Review. Journal of Thermal Biology, 34: 109-119.

Dillon M y Frazier M. 2006. Drosophila melanogaster locomotion in cold thin air. The Journal of Experimental Biology, 209: 364-371.

Ellenberg H. 1979. Man's Influence on Tropical Mountain Ecosystems in South America: The Second Tansley Lecture. The Journal of Ecology, 67(2): 401-416.

Ferreira LB y Tidon R. 2005. Colonizing potential of Drosophilidae (Insecta, Diptera) in environments with different grades of urbanization. Biodiversity and Conservation, 14: 1809-1821.

Figuero ML, Rafael V y Céspedes D. 2012. Grupo Drosophila asiri (Diptera, Drosophilidae) un nuevo grupo de especies andinas, con la descripción de dos nuevas especies y la redescripción de Drosophila asiri. Iheringia, Série Zoologia, 102(1): 33-42.

Figuero ML y Rafael V. 2011. Dos nuevas especies del grupo Drosophila onycophora, (Diptera, Drosophilidae) en los bosques de Polylepis de Papallacta, Pichincha, Ecuador. Iheringia, Série Zoologia, 101(4): 342-349.

Gottschalk MS, De Toni DC, Valente VLS y Hofmann PRP. 2007. Changes in 
Brazilian Drosophilidae (Diptera) assemblages across an urbanisation gradient. Neotropical entomology, 36(6) Londrina.

Grez AA y Zaviezo T. 2002. Efectos inmediatos de la fragmentación del hábitat sobre la abundancia de insectos en alfalfa. Ciencia $e$ Investigación Agraria, 29(1): 29-34.

Hoffmann AA. 2010. Physiological climatic limits in Drosophila: patterns and implications. The Journal of Experimental Biology, 213: 870-880.

INAMHI. 2009. Datos de pluviosidad en la estación AMAZNOR (Papallacta). Ecuador. Datos proporcionados por el Dr. Raúl Quiñones S.

James A, Azevdo R y Partridge L. 1997. Genetic and Environmental Responses to Temperature of Drosophila melanogaster from a Latitudinal Cline. Genetics, 146: 881-890.

Kessler M. 2006. Bosques de Polylepis. Botánica Económica de Los Andes Centrales. Ed.: M. Moraes R., B. Øllgaard, L. P. Kvist, F. Borchsenius y H. Balslev. Universidad Mayor de San Andrés, La Paz. p.110-120.
Magurran AE. 2004. Measuring Biological Diversity. Blackwell. Oxford. 256 pp.

Mateus RP, Buschini, M.L.T. y Sene F.M. 2006. The Drosophila community in Xerophytic vegetations of the upper Parana-Paraguay River Basin. Brazilian Journal of Biology, 66(2B): 719-729.

Medeiros HF y Klaczko LB. 2004. How Many Species of Drosophila (Diptera, Drosophilidae) remain to be described in the forest of São Paulo, Brazil? Species List of Three Forest Remnants. Biota Neotropica, 4(1).

Meyer CL y Sisk TD. 2001. Butterfly Response to Microclimatic Conditions Following Ponderosa Pine Restoration. Restoration Ecology, 9(4): 453-461.

Mittermeier RA, Gil PR y Mittermeier CG. 1997. Megadiversity: Earth's Biologically Wealthiest Nations. Conservation International, Cemex.

Rafael V. 2007. Drosophila malerkotlina y Zaprionus indianus (Diptera, Drosophilidae) invaden poblaciones ecuatorianas de Drosophila. Revista Ecuatoriana de Medicina y Ciencias Biológicas, 28(1 y 2): 30-43.

Kessler M. 2002. The "Polylepis Problem": Where do we stand? Ecotropica, 8: 97-110.
Rafael V y Vela D. 2000. Drosophila distribution in Ecuador. Drosophila Information Service, 83: 85-88. 
Shorrocks B. 1972. Invertebrate Types. Vela D y Rafael V. 2005. Catorce nuevas Drosophila. Ginn \& Company. Pergaespecies del género Drosophila mon Press, London, England. 144 pp. (Diptera, Drosophilidae) en el bosque húmedo montano del Volcán

Tidon R. 2006. Relationships between Pasochoa, Pichincha, Ecuador. Revista Drosophilids (Diptera, Drosophilidae) and the environment in two Ecuatoriana de Medicina y Ciencias Biológicas, 27: 27-41. contrasting tropical vegetations. Biological Journal of the Linnean So- Vela D y Rafael V. 2003. Estudios sobre la ciety, 87: 233-247. Diversidad del Género Drosophila (Diptera, Drosophilidae) en el

Vander LindeKy Sevenster J.2002.Drosophila diversity over a disturbance gradient. Proceedings Experimental and Applied Entomology, 13: 51-56. Bosque Pasochoa de la provincia de Pichincha-Ecuador. Revista de la Pontificia Universidad Católica del Ecuador, 71: 117-127. 